\title{
1 Position- and scale-invariant object-centered spatial selectivity in monkey 2 frontoparietal cortex dynamically adapts to task demand
}

3 Bahareh Taghizadeh ${ }^{1,2}$, Ole Fortmann ${ }^{1,3}$, *Alexander Gail ${ }^{1,3,4,5}$

$4 \quad{ }^{1}$ Cognitive Neuroscience Laboratory, German Primate Center, Göttingen, Germany

$5 \quad{ }^{2}$ School of Cognitive Science, Institute for Research in Fundamental Sciences (IPM), P.O. Box 19395-5746, Tehran, 6 Iran

$7 \quad{ }^{3}$ Faculty of Biology and Psychology, University of Göttingen, Göttingen, Germany

$8 \quad{ }^{4}$ Bernstein Center for Computational Neuroscience, Göttingen, Germany

$9 \quad{ }^{5}$ Leibniz ScienceCampus Primate Cognition, Göttingen, Germany

10

11 Corresponding author: Alexander Gail (agail@gwdg.de)

12

13 Keywords: parietal cortex, premotor cortex, dorsal stream, allocentric reference frame, reach movement, size14 invariance.

16 Authors' contribution: B.T. and A.G. designed and implemented the experiment, and collected the data. B.T. and 17 O.F. analyzed the data. B.T., O.F. and A.G. discussed the results and wrote the paper. 


\section{Abstract}

31 Humans utilize egocentric and allocentric spatial information to guide goal-directed movements.

32 Egocentric encoding is a well-known property of brain areas along the dorsal pathway. We ask if

33 dorsal stream reach planning areas like parietal reach region (PRR) and dorsal premotor cortex

34 (PMd) also encode object-centered (allocentric) information. During two consecutive instructed

35 delay periods, rhesus monkeys first memorized an object-relative target position and then

36 planned a reach to this position after the object re-occurred at variable positions with potentially

37 different size. In both areas, we find predominant object-centered encoding during visual

38 memory, which is invariant to object position and object size, and predominant egocentric

39 encoding during reach planning. Such dynamic transition from allo- to egocentric encoding

40 within individual dorsal stream areas contrasts the idea of task-independent functional

41 segregation between processing pathways. Instead, demand-specific local computations might

42 facilitate spatial cognition in dynamic environments to facilitate motor planning towards objects

43 changing their location.

\section{Significance statement}

45 Human and non-human primates interact with their environment by manipulating objects. This

46 requires planning and executing reaches to transport the hand to object-relative positions close to

47 or on the object. Previous studies showed that frontal and parietal lobe areas encode reach goals

48 in coordinate systems anchored to different parts of the subjects' body, such as hand position or

49 gaze direction, and modulated by pose. We show that neurons in the same areas can encode

50 object-centered allocentric spatial information, independent of object location and object size, or

51 egocentric information, depending on dynamically changing task demands. Such dynamic

52 adaptation seems inconsistent with a functional segregation for ego- and allocentric encoding

53 between processing streams. 


\section{Introduction}

55 Allocentric (subject-independent) spatial cognition is a fundamental skill supporting navigation

56 (Moser et al., 2008), spatial judgment (Aagten-Murphy and Bays, 2019; Galati et al., 2000;

57 Vallar et al., 1999; Zaehle et al., 2007), and goal-directed movement behavior (Byrne et al.,

58 2010). Since primary spatial sensory inputs are egocentric, e.g. determined by visual retinotopy

59 and tactile somatotopy, allocentric encoding must be the result of neural computation. Yet, brain

60 regions, especially the roles of ventral versus dorsal stream processing, and neurocomputational

61 mechanisms of allocentric encoding are still under debate (Chen and Crawford, 2020; Deneve

62 and Pouget, 2003; Filimon, 2015). Neurophysiology data at the single neuron level in the context

63 of allocentric goal-directed reaching is lacking. Here we ask if object-centered encoding can be

64 observed during goal-directed reach planning in frontoparietal areas of the dorsal stream, i.e.,

65 areas which are mostly associated with egocentric frames of reference, e.g., (Battaglia-Mayer et

66 al., 2003; Bremner and Andersen, 2014; Chang and Snyder, 2010).

67 Natural reaches typically are directed towards physical objects. Geometrical features such as

68 shape and size of that object should be incorporated in movement planning to successfully direct

69 the hand towards a suited part of the object. We may pick up a stick at different positions along

70 its length, depending on the intended use. Such object-oriented reach goal may only depend on

71 object properties and hence can be defined independently of the spatial positioning of the object

72 relative to the subject. This would mark a case of reach-associated, yet object-centered

73 allocentric spatial processing for the localization of the target position of the reach. Additionally,

74 the inherently egocentric changes in body-configuration needed to accomplish the reach will

75 become relevant for planning and implementing it. It is an open question if dynamically

76 changing allo- and egocentric spatial cognitive demands for planning goal-directed movements

77 are fulfilled in separate specialized brain regions, or if the same local neural network within an

78 area can reflect both types of encoding.

79 Human neuroimaging studies on allocentricity in the context of perceptual judgment tasks (with

80 verbal or button-press responses) suggest an anatomical segregation, where the dorsal stream

81 'vision-for-action' processing is predominantly egocentric and the ventral stream 'perceptual'

82 and 'object-recognition' processing encompasses also allocentric representations (Galati et al., 
2000; Neggers et al., 2006). Yet, it may be task dependent which processing predominates and it

84 has been shown that allocentric spatial judgments can activate networks comprising areas of both dorsal and ventral streams (Committeri et al., 2004; Zaehle et al., 2007). Human neuroimaging studies on target localization partly also indicated activation of overlapping regions of the frontoparietal network for planning and guiding goal-directed reach and saccade movements relative to egocentric and allocentric references (Chen et al., 2018, 2014, 2011; Thaler and Goodale, 2011a, 2011a, 2011b). However, the role of dorsal stream in allocentric processing is not fully clear from imaging studies. While allocentric compared to egocentric encoding of reach target distance and direction led to higher blood-oxygen-level-dependent (BOLD) activity in dorsal premotor cortex (PMd) and right posterior intraparietal sulcus of human subjects (Thaler and Goodale, 2011c), others did not find such preference along the dorsal stream (Chen et al., $2018 ; 2014)$.

Single neuron recordings in monkeys revealed how sensory information is transformed into motor goal information within the frontoparietal network during reach planning (Cui and Andersen, 2011). Neurons in monkey posterior medial intraparietal cortex (parietal reach region, PRR) and PMd are selective for the spatial location of visual cues indicating the target for a reach, but also cue-independent reach goals (Cisek and Kalaska, 2005; Crammond and Kalaska, 1994; Gail et al., 2009; Gail and Andersen, 2006; Hwang and Andersen, 2012; Riehle and

101 Requin, 1989; Snyder et al., 1997; Westendorff et al., 2010; Wise et al., 1997). In monkeys that

102 aim their reach at visually instructed positions, while controlling gaze independently, different

103 frames of reference have been described, all of which are egocentric. Many studies reported 104 predominant gaze-centered (Batista et al., 2007, 1999; Bremner and Andersen, 2014; Buneo et 105 al., 2002; Chang and Snyder, 2010; Cohen and Andersen, 2002; Marzocchi et al., 2008) or 106 predominant hand-centered (Bremner and Andersen, 2012; Caminiti et al., 2015, 1991, 1990; 107 Johnson et al., 1996) encoding of reach goals. Encoding within these areas is typically not 108 exclusive but rather intermediate with mixed (Chang and Snyder, 2012; McGuire and Sabes, 109 2011) and more complex selectivities (Pesaran et al., 2006), also found in humans (Zhang et al., 110 2017).

111 The prevailing view of dorsal stream processing that emerged from these neurophysiological 112 findings is that different visual and somatosensory inputs, which are of egocentric nature, 
113 become integrated in the parietal multisensory association cortex to compute reach-relevant

114 spatial information in different egocentric reference frames. Different anatomical nodes have a

115 predominance for reference frames being centered on different body parts (Batista et al., 1999;

116 Bremner and Andersen, 2012; Chang and Snyder, 2012, 2010), and the result of such

117 feedforward integration (Deneve and Pouget, 2003; Zipser and Andersen, 1988) is supposed to

118 be fed to more motor-related areas in the frontal lobe. Yet, if also allocentric spatial cognitive

119 processing in the context of goal-directed reaching exists in parietal cortex is unclear, since

120 single-cell electrophysiology studies directly comparing egocentric and allocentric reference

121 frames during reach planning do not exist.

122 Here we directly compare posterior medial intraparietal cortex (parietal reach reagion, PRR) and

123 dorsal premotor cortex (PMd) at the single neuron level in rhesus monkeys in an object-centered

124 allocentric reach task that sequentially mandates spatial target memory and reach planning. Two

125 experiments tested position- and size-invariant object-centered encoding. In contrast to the

126 prevailing view, we report that neurons in PRR and PMd encode cue and reach target in object-

127 centered as well as egocentric reference frame and the predominating reference frame in both

128 areas is dynamically adjusted to the cognitive needs. 


\section{Results}

130 Two male rhesus monkeys (Macaca mulatta) were trained to perform memory guided reaches 131 towards variable positions on an elongated object. The object had variable position relative to the 132 animal, allowing to dissociate object-centered (allocentric) locations from body-centered 133 (egocentric) reach goal locations (Fig. 1). Two instructed delays allowed separately investigating 134 spatial frames of reference during visual memory and reach planning, respectively.

\section{Figure 1: Object-based reach planning task}

(a)

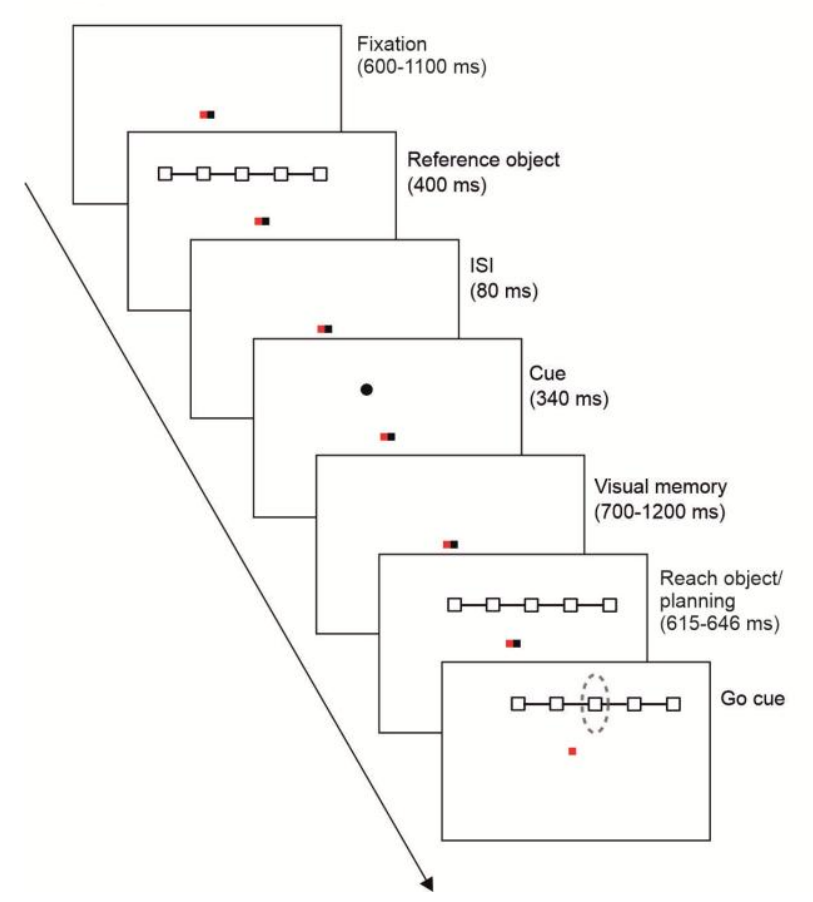

(b) Position invariance (Exp-l)

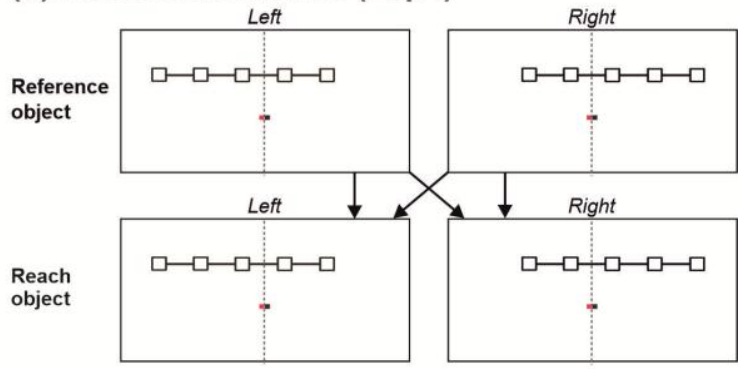

(c) Size invariance (Exp-II)

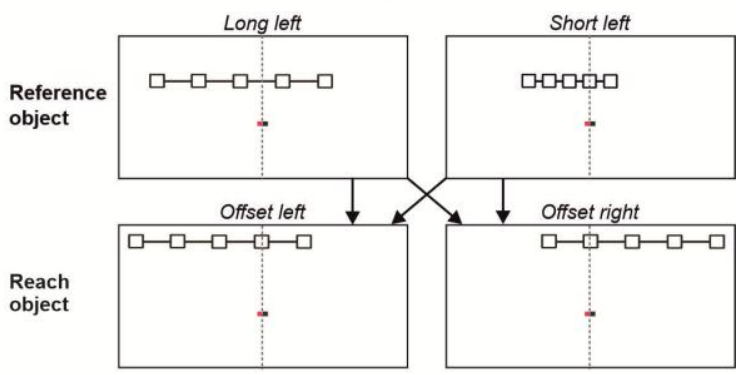

(a) Time course. After acquiring and holding successful ocular fixation of the central red square and touching of the white square during baseline, an array of five interconnected boxes (reference object) is presented randomly to the left or right of the screen center. The reference object is followed by a brief cue presentation, located at one of the five box positions (target box), and a visual memory period after which the array of boxes is presented again (reach object), randomly to the left or right of the screen center. During the following delay (movement planning period) the monkey has to maintain ocular fixation and withhold arm movement, thereby keeping body-, hand- and gaze-centered frames of reference aligned with the screen center. Disappearance of the hand fixation stimulus (Go signal) permits the monkey to reach to the memorized target box. The target box is defined by the position of the box that was cued on the reference object in object coordinates. Time spans of individual trial periods as depicted in the labels indicate the range of uniformly distributed random durations. (b) Position invariance (Exp-I). Reference and reach object each are presented at fixed eccentricities randomly to the left and right of the screen center. Left and right offsets are uncorrelated, making the position of the reach object unpredictable and in 50\% congruent and 50\% incongruent to the reference object. The horizontal offset between left and right object center corresponds to the inter-box distance, so that left and right object location overlap in four boxes. This results in a total of six possible egocentric cue/reach target locations on the screen (Supplementary Fig 1). In Exp-I, reference 
and reach objects are always the same size and also otherwise visually identical. (c) Size-invariance (Exp-II). In half of the trials the reference object had the same length as in Exp-I (example "long left" in upper row left) and in the other trials was half as long (example "short left" in upper row right). Again, reference and reach object each were presented randomly to the left or right of the screen center (Supplementary Fig 1). In Exp-II, horizontal offsets of reference and reach object differed in size and an additional vertical offset was introduced such that reference and reach object were incongruent in all trials (see Methods for details).

135 The animals memorized the location of a briefly flashed peripheral visual cue at one of five 136 positions on an elongated visual object (Fig. 1a; reference object) to later reach towards this on137 the-object position irrespective of object location on the screen (reach object). The egocentric 138 cue positions varied independently of their object-centered positions since the reference object 139 was presented with a left or right offset relative to the screen center, randomly in each trial (Fig.

140 1b). After a first delay period (visual memory), the object re-appeared as reach object, again 141 randomly with a left or right offset. After a second delay (reach planning), the monkeys had to 142 reach to the previously cued target position on the object. The location of the reference object 143 was not predictive for the location of the reach object. In experiment I (Exp-I; Fig. 1b), reference 144 and reach object were visually identical but their location could be shifted to test for position145 invariant (=object-centered) encoding. In experiment II (Exp-II, Fig. 1c), additionally, the size 146 could vary between reference and reach objects to test for position- and scale-invariant object147 centered encoding (see Methods for details).

\section{Object-centered vs egocentric encoding hypothesis}

149 In Exp-I, we asked if the fronto-parietal network encodes the location of the cue (visual memory) 150 and the associated reach goal (reach planning) either in an object-centered or egocentric 151 reference frame. Fig. 2a illustrates reference-object-left and reference-object-right selectivity 152 profiles for two hypothetical neurons, representing object-centered and egocentric reference 153 frames, respectively. In object-centered encoding, spatial selectivity of the neuron only depends 154 on the position relative to the object. Thus, such neuron would show the same pattern of 155 selectivity to different boxes on the object in object-left and -right conditions. Consequently, in 156 body-centered screen coordinates (Fig. 2a, left) this corresponds to a shift of the selectivity 157 profile that matches the object's shift, while the shape of the profile stays the same. 
bioRxiv preprint doi: https://doi. org/10.1101/2022 0126.477941. this version posted January 28,2022 . The copyright holder for this preprint (which was not certified by peer review) is the author/funder, who has granted bioRxiv a license to display the preprint in perpetuity. It is made available under aCC-BY-ND 4.0 International license.

\title{
Figure 2: Object-centered and egocentric reference frames
}

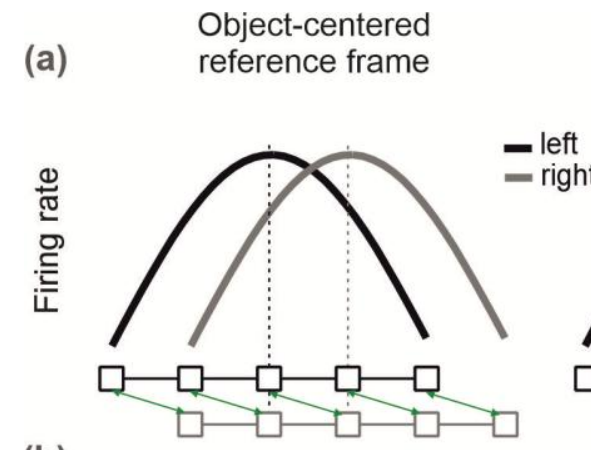

\author{
Egocentric \\ reference frame
}

(b)

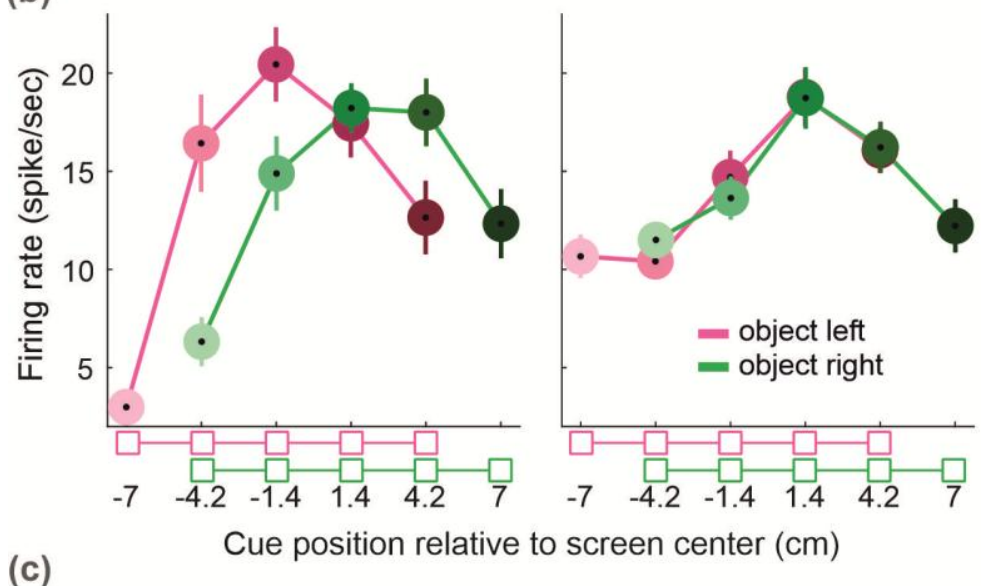

(c)
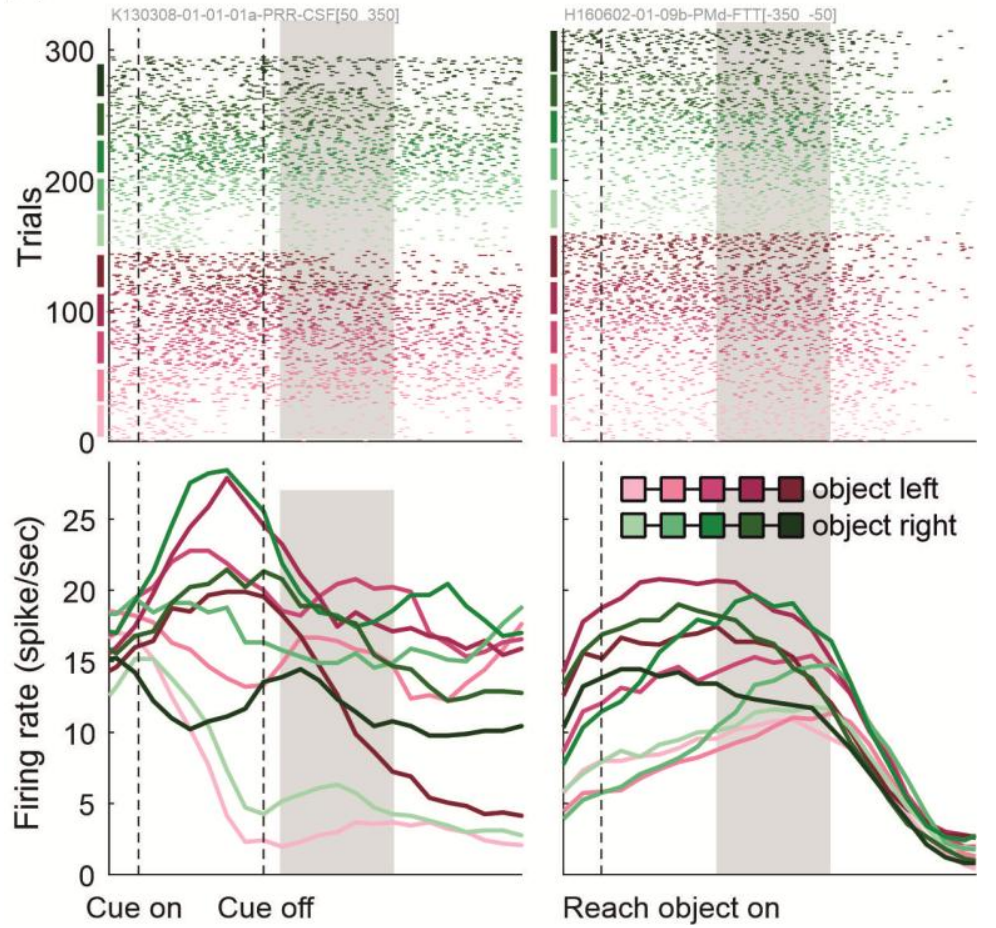

(a) Hypothetical object-centered and egocentric single unit responses. The object-centered hypothesis (left column) predicts that a neuron keeps the same selectivity profile for different cue/target locations on the object, 
independently of the object location on the screen (=relative to the body). When analyzed as function of screen position, this would result in a shift of the profile together with the object. The egocentric hypothesis (right column) predicts that the selectivity profile is a function of the cue/target location relative to the body and not relative to the object. When comparing object-left and object-right profiles, the neuron shows the same response level for target positions overlapping in screen space, but different activity for non-overlapping boxes. Shifting of the object in this case would mean sampling a different part of the egocentric selectivity profile. (b) Examples of single unit selectivity profiles from Exp-I. An example single unit shows object-centered encoding (left column) of the cue during early visual memory period, 50 to $350 \mathrm{~ms}$ after cue offset (shaded time window in (c)). Another example shows egocentric encoding (right column) of the target during planning period, 350 to $50 \mathrm{~ms}$ before movement onset. The curves show mean firing rate across same-condition trials, error bars indicate SEM. Light/dark color shades correspond to the boxes on the object). (c) Raster plot and PSTH of example units. Left and right columns show raster plots (top row) and peri-stimulus time histograms (PSTHs, bottom row) from respective example units in (b). In the raster plot, every row is one trial and trials are sorted and grouped according to the on-the-object positions. The PSTHs below show spike frequencies (Gaussian kernel smoothing with $50 \mathrm{~ms}$ standard deviation) averaged across trials with identical object and on-the-object cue/target positions.

160 On the other hand, if an ideal neuron encodes the cue in an egocentric coordinate system, the 161 selectivity will only depend on the egocentric location of the cue regardless of the object 162 location. By shifting the object on the screen, one would sample different segments of an 163 egocentric selectivity profile. Therefore, when comparing the object-left and object-right 164 selectivity profiles, an egocentric neuron would show the same activity for positions with 165 corresponding egocentric locations (Fig. 2a, right). This logic applies to cue encoding relative to 166 reference object and reach goal encoding relative to the reach object.

167 Single units in PRR and PMd encode location of the cue and reach goal in object-centered as well as in egocentric reference frames

169 Monkeys $\mathrm{K}$ and $\mathrm{H}$ performed the task with $72.15 \% \pm 1.00 \%$ and $87.92 \% \pm 1.20 \%$ average 170 success rate across sessions. We recorded 100 and 107 single units in PRR and PMd, 171 respectively, from monkey $\mathrm{K}$, and 56 and 57 in PRR and PMd, respectively, from monkey $\mathrm{H}$. 172 From monkey K, 71 (71\%) of neurons in PRR and 77 (72\%) in PMd were included in the 173 following analysis, from monkey H, 30 (54\%) in PRR and 32 (56\%) in PMd (see Methods).

174 Since data from the two monkeys yielded corresponding results, throughout, we report the result

175 from combining the two monkeys' data unless mentioned otherwise.

176 During the visual memory period, selectivity profiles of a subset of cells in both areas were 177 consistent with encoding of the cue location in an object-centered reference frame (Fig. 2b-c, left 178 panels). The selectivity profile of this example neuron is independent of the screen-location of 179 the object, hence object-centered. Single units with object-centered selectivity were found in 180 both areas PRR (shown) and PMd of both monkeys. 
181 Selectivity profiles of other units in the same areas and in both animals resembled egocentric

182 encoding of the cue location (Fig. 2b-c, right panels). This example unit showed identical neural

183 response strengths when overlapping boxes (i.e. egocentrically corresponding cue location) were

184 cued with the object being located either left or right. Neural response strengths differed for non-

185 overlapping boxes, i.e. when new locations relative to the body of the animal were sampled due

186 to the new object location.

187 During the movement planning period, we also observed that selectivity profiles of some neurons

188 resembled encoding of the reach goal in the object-centered reference frame and others in an 189 egocentric reference frame (data not shown). As is not surprising for mixed encoding, during 190 both the visual memory period and reach planning, selectivity patterns of most neurons only 191 partly match the idealized patterns shown in Fig. 2a. Often neurons show more complex patterns 192 with ambiguous or mixed object-centered and egocentric selectivity. Simple examples of 193 ambiguity are linear selectivity profiles, in which case object-left and object-right tuning can 194 result either from a horizontal shift or a vertical (firing rate) offset between both conditions (not 195 shown).

196 To respect the continuous spectrum reflected in the observed mixed selectivity not only across 197 areas or units, but also within individual units, we did not attempt to categorize the spatial 198 selectivity of single units as binary object-centered or egocentric classes. Yet, the existence and 199 gradual tendencies for either encoding scheme at the neural population level nevertheless can be 200 quantified and might differ between brain areas or cognitive states and be informative about 201 underlying computations.

202 Predominant reference frame changes from object-centered during visual memory to 203 egocentric during motor planning in PRR and PMd

204 During the late visual memory period, object-centered encoding of the cue location 205 predominated across the neural population in both PMd and PRR. We quantified population206 level predominance of either encoding scheme over the course of the trial in two ways: first, by 207 computing a position invariance (PI) index, which is based on correlations of spatial selectivity 208 profiles between conditions with different object positions (see Methods); second, with 209 population decoding based on a cross-conditional classifier. 
210 Positive values of the average (across neurons) PI during the memory period indicate 211 predominant object-centered encoding (Fig. 3a). Once the location of the reach object is 212 provided, the egocentric reach goal location becomes known to the monkeys for movement 213 planning. After this reach object onset, the average PI shifted within 300-500ms, from 214 predominant object-centered to predominant egocentric spatial selectivity in both brain areas 215 (Fig. 3a). The single unit analysis allows to test if the shift of the PI distribution at the population 216 level is due to selective activity of two different neuronal subpopulations in memory and 217 planning periods ("recruitment"), or rather the consequence of a consistent shift in preferred 218 encoding of the individual neurons ("re-coding"). When comparing visual memory and 219 movement planning periods (Fig. 3b), the PI values cluster in the lower right quadrant and the 220 angular density distribution is unimodal, as expected for the re-coding hypothesis. There is no 221 indication of a clustering along the main axes and a corresponding bimodal angular density 222 distribution, as would be expected for the recruitment hypothesis (Hartigan's dip test; PRR $223 \mathrm{p}=0.84$; PMd $\mathrm{p}=0.85$ ). This re-coding pattern suggests that in PRR and PMd, the population of neurons contributing to either predominant reference frame in the two different periods mostly 225 overlap. There is a weak but statistically significant negative correlation between PI of neurons 226 in late memory period and movement planning period in PRR but not PMd (Pearson's 227 correlation coefficient; PRR $r_{p}=-0.24, p=0.015, P M d p=0.3$ ). This suggests that at least in 228 PRR, on average, units with stronger object-centered preference in late memory tend to be more 229 strongly egocentric in the later planning period.

230 Second, we used neural population decoding to quantify the predominance of either reference 231 frame. For this, we applied cross-conditional classification, and characterized generalization 232 errors. Classifiers were trained to distinguish the five positions on the object using only trials 233 where the object was on one side and tested its performance on trials where the object was on the 234 other side. Predominant egocentric or object-centered neural reference frames, respectively, then 235 predict characteristic classification patterns. High classification accuracy suggests an object236 centered population encoding since each position on the object is decoded correctly irrespective 237 of the object location relative to the body. Egocentric encoding instead would introduce 238 systematic misclassification, namely a shift by one position off the main diagonal in the confusion matrix (Fig. 3d, top row). 


\section{Figure 3 Population response with varying object position (Exp-I)}

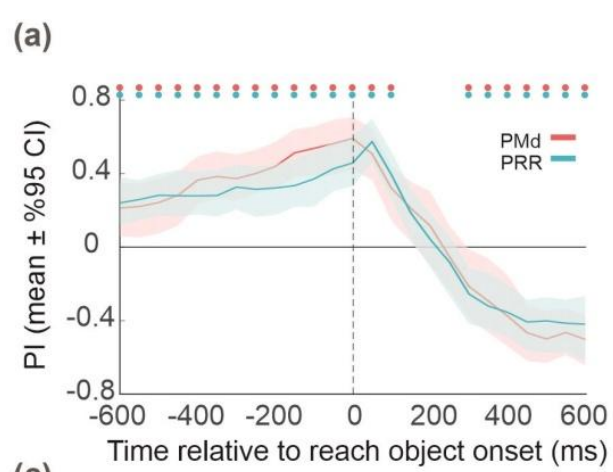

(c)

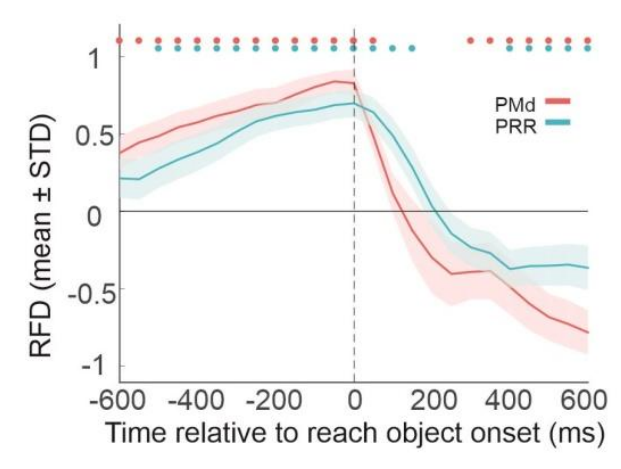

(b)

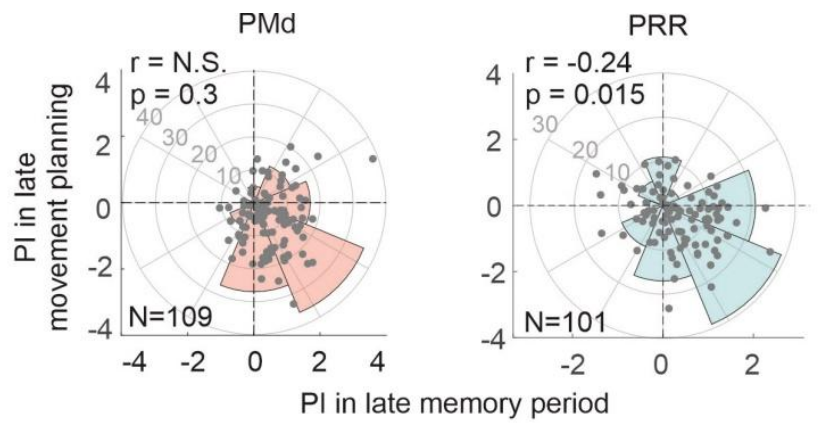

(d)

Training with left object Training with right object

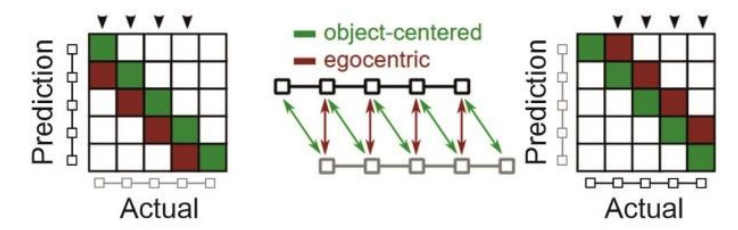

Training with left object Training with right object

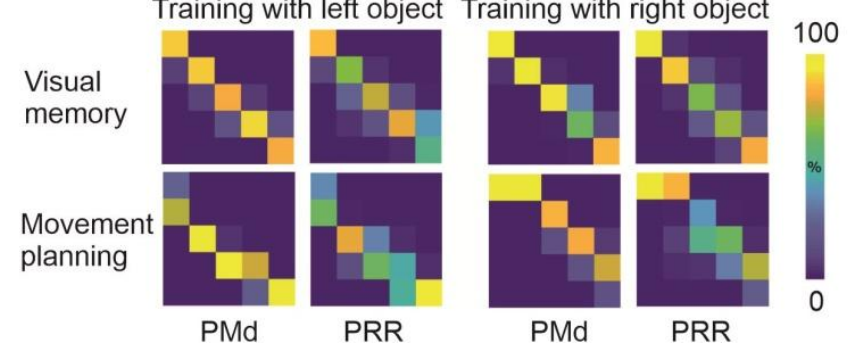

(a) Preferred reference frame across neuronal population. Position Invariance (PI) measure across the population of 101 PRR (blue) and 109 PMd (red) single units (300 ms time bins, sliding by $50 \mathrm{~ms}$ ). Positive and negative PI values indicate dominant object-centered and egocentric encoding, respectively. In the visual memory period (before reach object onset), object-centered encoding gained higher weight across the population, whereas reach goal locations (after reach object onset) were dominantly encoded in an egocentric reference frame. Dots at the top indicate significant deviation from zero (t-test, $\mathrm{p}$ values corrected for multiple comparison across time bins using False Discovery Rate correction (see Methods)) (b) Reference frame in memory vs movement planning period. PI values of individual units (dots in scatter plot) in late memory (300 ms before reach object onset) vs late reach planning period (300-600 ms after reach object onset) are shown. Angular densities across the population of neurons do not indicate deviations from a unimodal distribution (Hartigan's dip test: PRR $p=0.84$; PMd $p=0.85$ ). $r$ and $\mathrm{p}$ indicate Pearson's correlation coefficient and its significance level. (c) Relative difference of reference frame decoding (RFD). Positive and negative RFD values indicate dominant classification of object-centered and egocentric positions, respectively similar to the time courses from the tuning analysis in (a). Dots at the top indicate significant deviation from zerp; $\mathrm{p}$ values corrected for multiple comparison across time bins using False Discovery Rate correction (see Methods). (d) Hypothetical and actual confusion matrices. In RFD, a decoder (5way classifier) trained with the trials with the object on one side of the screen that is able to accurately classify the object-centered position of the target box when the object is on the other side of the screen. In the hypothetical confusion matrix (top row) these trials fall on the main diagonal (green). An egocentric encoding, instead, would introduce a systematic misclassification of the data, resulting in diagonals that are one off the main diagonal to the left or right (red), depending on which side is used for training the classifier. Arrows on top of the matrix mark the task conditions that are included in the calculation of the RFD values, as they have a distinct egocentric and objectcentered representation (see Methods). Bottom row shows actual confusion matrices. During the late memory period object-centered positions are classified accurately. During late movement planning, a shift off the diagonal indicates classification of egocentric positions in both areas. 
From monkey K, 83-84 (83-84\%) of neurons in PRR and 99-101 (93-94\%) in PMd were included in the decoding analysis based on the number of available trials per condition (see

242 Methods), from monkey H, 39-41 (70-73\%) in PRR and 43-45 (75-79\%) in PMd. There is a 243 small variability in the number of neurons between conditions because the exact number of 244 available trials depends on the condition that was used for training the decoder. When tested on 245 the same data as used for training (iso-conditional), the classifiers showed high cross-validated 246 classification accuracy of $95 \%$ for PMd and $80 \%$ for PRR on average over time bins and trial 247 subsamples (data not shown). To test for the predominant reference frame, we computed the 248 difference between the percentage of test trials classified in accordance with the object-centered 249 hypothesis and the percentage classified in accordance with the egocentric hypothesis during the 250 cross-conditional decoding (reference frame difference RFD; see Methods). The RFD (Fig. 3c) 251 and confusion matrices (Fig. 3d, bottom row) show that during the visual memory period the cue 252 position is predominantly classified in accordance with an object-centered reference frame (RFD $253>0$; PRR $\mathrm{p}<0.01$, PMd $\mathrm{p}<0.01)$. Then there is a transition to classifying positions 254 predominantly in an egocentric reference frame $(\mathrm{RFD}<0$; $\mathrm{PRR} \mathrm{p}<0.01$, $\mathrm{PMd} \mathrm{p}<0.01)$ in the 255 late movement planning phase.

Single units scale the width of their selectivity profile with object size in PRR and PMd

257 Our results from Exp-I suggest a predominant object-centered encoding of the cue location 258 during the memory period across the neuronal population. In Exp-II, we asked if the fronto259 parietal network can encode on-the-object locations in an object-centered manner also for 260 variable object size. Real-life objects might change their position not just within a fronto-parallel 261 plane, but also in depth, thereby changing their visual size; or, they might exist in different-size 262 variants, like hammers for different purposes. Hence, ideal object-centered encoding of on-the263 object locations predicts that selectivity profiles of neurons should be characterized only by the 264 relative positioning of the cue and the object, irrespective of object location (origin) and object 265 size (scale), i.e., by position-invariant and size-invariant object-centered selectivity. In Exp-II, 266 the monkeys saw a long or a short reference object, in randomly interleaved trials, relative to 267 which they had to memorize the cue location (Fig. 1c). In order to preserve object-centered 268 position information, one would expect a compression of the selectivity profile (in the egocentric 269 screen space) for object-short compared to object-long trials (Fig. 4a, left). In contrast, 
270 egocentric encoding would result in sampling of a reduced range of the selectivity profile in 271 object-short trials (Fig. 4a, right).

272 For Exp-II, we recorded 67 and 53 single units in PRR and PMd, respectively, from monkey K; 27336 and 45 single units in PRR and PMd from monkey H. We identified 51 (76\%) and $46(87 \%)$ 274 active units in PRR and PMd from monkey K, 19 (53\%) and 20 (44\%) from monkey H, which 275 were included in the following analysis. Monkeys $\mathrm{K}$ and $\mathrm{H}$ performed the task with $63.94 \% \pm$ $276 \quad 0.88 \%$ and $81.70 \% \pm 2.50 \%$ average success rate.

277 Neurons in PRR and in PMd of both monkeys revealed examples of size-invariant object278 centered encoding during visual memory. Fig. $\mathbf{4 b}$ and $\mathbf{4 c}$ show an example unit where the object279 short response profile fits the prediction of the object-centered hypothesis in the sense that it 280 represents a duplicate of the long-object selectivity profile compressed by the factor that short 281 and long object differ in length. The example neuron showed the same selectivity to long and 282 short reference objects, respectively, when they were presented on the left of the screen center 283 (not shown). In other words, only the relative on-object position determined the response, not the 284 location of the object on the screen nor the size of the object. As in Exp-I, also in Exp-II we 285 observed units with more complex selectivity profiles which either do not or only partly fit the 286 object-centered or the egocentric hypothesis (Supplementary Fig. 2). We tested for size 287 invariance only during the visual memory period, not during movement planning, since Exp-I 288 had shown dominant object-centered encoding during the memory period only. Consequently, in 289 Exp-II, short objects were only used as reference objects, not as reach objects.

290 In order to assess the contribution of position- and size-invariant encoding at the neural 291 population level, we again computed the correlation measure and the decoding measure. For the 292 correlation measure, we computed PSI for each neurons selectivity profiles (see Methods). The 293 distribution of PSI across units (Fig. 4e) shows that different units scale their selectivity profile 294 to different extent, evident from the range of PSI values across the population. However, on 295 average, size-invariance predominated (signed-rank test, PRR $\mathrm{p}=7 \times 10^{-9}, \mathrm{PMd} \mathrm{p}=10^{-9}$ ), 296 consistently across areas. 


\section{Figure 4 Size-invariant object-centered encoding (Exp-II)}

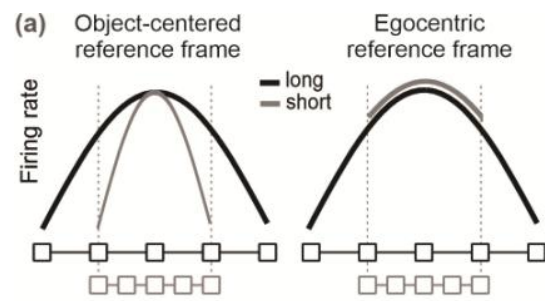

(b)

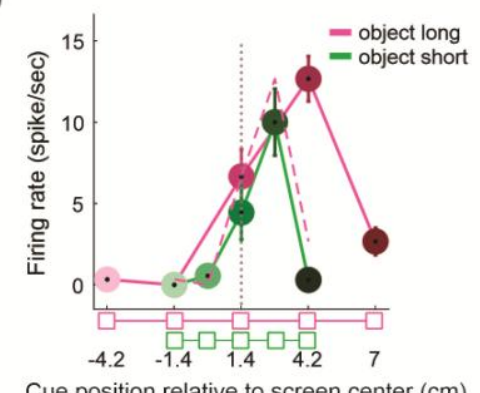

(c)

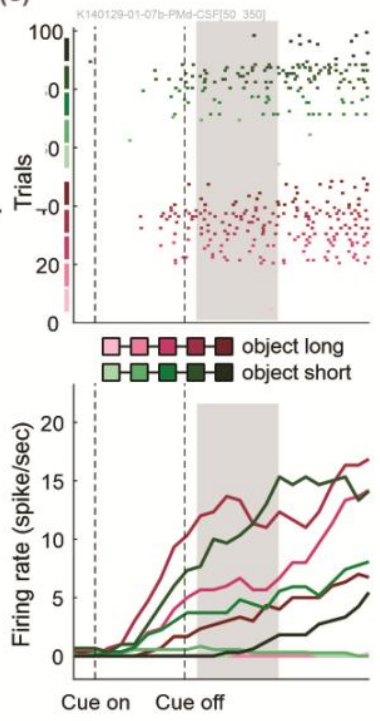

(d)

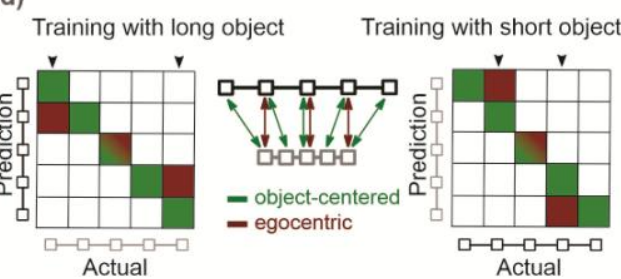

(e)

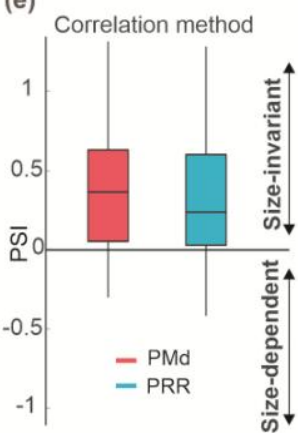

(f)

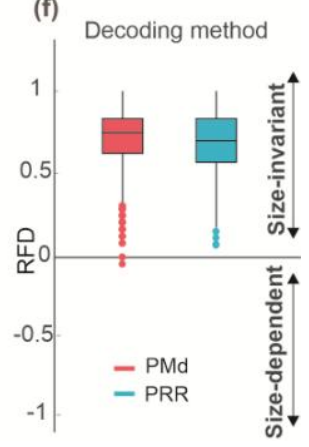

(a) Hypothetical size-dependent and -invariant single unit responses. Selectivity profiles of ideal objectcentered size-invariant (left) and egocentric size-dependent (right) neuronal encoding. The size-invariance hypothesis predicts that the selectivity profile scales with the object size, such that the profile to different locations on the object is the same irrespective of object size. The egocentric hypothesis predicts the same response level for positions with identical egocentric locations, thereby sampling less horizontal extend when objects are small. (b-c) Example unit from Exp-II. Activity of an example unit when short and long reference objects were presented at the right side of the screen center. Object-centered size-invariant encoding is identified by a horizontally scaled selectivity profile. The dashed curve represents the ideal object-centered scaling of the selectivity profile for shortobject trials, predicted from the selectivity profile observed in long-object trials, assuming that the width of the activity profile was scaled with the size of the object and relative to center of the object. The actually measured profile in this example precisely matched the prediction except for a small reduction in response gain by about $10 \%$. If normalizing to the maximal response, result and prediction match exactly (not shown). All other settings are as in Fig. 2b-c. (d) Hypothetical confusion matrices. In RFD, a decoder (5-way classifier) trained with the trials with one object size that is able to accurately classify the object-centered position of the target box when the object is of the other size. In the hypothetical confusion matrix these trials fall on the main diagonal (green). An egocentric encoding, instead, would introduce a systematic misclassification of the data, resulting in diagonals that are compressed compared to the main diagonal, vertically or horizontally (red), depending on which object size is used for training the classifier. Arrows on top of the matrix mark the task conditions that are included in the calculation of the RFD values, as they have a distinct egocentric and object-centered representation (see Methods). (e) Mostly positive Position and Size Invariance (PSI, see Method) values across the population of 66 PMd (red) and 70 PRR (blue) single units indicate predominant size-invariant neural selectivity in the last $300 \mathrm{~ms}$ of visual memory period (signed-rank test, PRR $\mathrm{p}<7 \times 10^{-9}$, PMd $\mathrm{p}<10^{-9}$ ). (f) Equivalently, positive RFD values indicate dominant classification of object-centered rather than egocentric positions, respectively. The box plots show median and the $75^{\text {th }}$ (top) and $25^{\text {th }}$ (bottom) percentiles, as well as the data range (whiskers)without putative outliers (dots; more distant from 25/75 percentiles than 1.5 times the respective interquartile range).

With the cross-conditional classification approach, we tested for generalization of the decoder between the long and short reference object conditions (see Methods). The cross-validated accuracy of the classifiers on the training data was high during the visual memory period, $88 \%$ 
302 in both areas the cue position predominantly can be decoded invariant to the size of the object

303 during the late visual memory period (RFD > 0; PRR $\mathrm{p}<0.01$, PMd $\mathrm{p}<0.01$ ).

\section{Discussion}

305 Reaching towards and grasping objects is a major way in which primates interact with their environment. Object-relative position information is important in guiding these movements.

307 Such allocentric alongside egocentric frames of reference provide a stable representation of 308 space which is robust to environmental dynamics and noise (Burgess, 2006). Having such 309 stability in spatial representations is crucial for goal-directed reach planning when, for example, 310 the reach goal is located on a moving object or when the object is temporarily occluded. Here we 311 showed, first, that object-centered spatial encoding is part of the mixed ego- and allocentric 312 neural selectivity found in the frontoparietal reach planning network when reach is directed 313 towards different on-the-object sites. Second, in both, PRR and PMd the predominant frame of 314 reference shifts according to cognitive demands in different epochs of the behavioral task. 315 During visual memory, when the cue-on-object location was the most parsimonious way of 316 maintaining the relevant spatial information, object-centered encoding predominated. During 317 movement planning, when the actual reach goal could be determined from the integration of the 318 memorized cue-on-object location and the now visible and stationary reach object location, 319 egocentric encoding predominated. Third, by providing evidence for size-invariant spatial 320 selectivity, we demonstrate that allocentric encoding not only means that the origin of the 321 reference frame is centered on the object, but also that the spatial scale scales with object-size.

322 Such size-invariant representation of object-related information so far was associated with 323 object-recognition tasks and mainly ventral stream processing (DiCarlo and Cox, 2007; Grill324 Spector and Weiner, 2014; Tacchetti et al., 2018), but not with dorsal stream movement 325 preparation.

326 For successful physical interactions, a geometrical representation of the object, including 327 features such as object size and on-the-object sites, is necessary for action planning. Additional 328 to pre-shaping the hand to fit the geometry of the object before grasping (Aglioti et al., 1995), a 329 proper object-relative placement of the hand is needed, e.g., to pick up a hammer at its handle 330 instead of its head. Correct object-relative hand positioning is relevant for successful completion 
331 of the object-associated action, and hence might differ from allocentric localization of reach 332 goals relative to a landmark with which one is not going to have direct physical interaction.

333 When localizing reach goals relative to a landmark, human imaging data suggest that spatial 334 processing along the dorsal stream is predominantly egocentric (Chen et al., 2014). In contrast, 335 when human subjects had to spatially judge reach goals based on geometrical features like 336 relative distance and direction of a remote pair of dots, the frontoparietal network was more 337 active for such allocentric movements compared to egocentric movements directed towards a 338 visual target (Thaler and Goodale, 2011c).

339 We see that either reference frame can dominate in the frontoparietal reach network. The 340 allocentric frame dominated while the animals had to memorize an on-the-object position to 341 generalize this geometric information to the time when the final object location on the screen 342 would become available. The egocentric frame dominated as soon as animals could plan an 343 according reach movement (Chen et al., 2014). While mixed egocentric reference frames have 344 been described before (Chang and Snyder, 2010; McGuire and Sabes, 2011), our findings further 345 suggest that predominant reference frames are not fixed properties in frontoparietal association 346 cortices. Instead, they reflect task-specific computations to achieve spatial transformation, 347 thereby not just giving variable weight to different egocentric sensory modalities (Bernier and 348 Grafton, 2010; Bremner and Andersen, 2014), but also including allocentric computations if 349 demanded by the task. The diversity of preference, which ranges from egocentric to object350 centered encoding across neurons in PRR and PMd provides high flexibility for the fronto351 parietal network for conveying spatial information to recipient areas in either of the two frames 352 of reference.

353 Overall, human imaging studies suggest partially overlapping networks for allocentric reach 354 movement planning (target memory) and egocentric guidance of movements. Yet, while (Chen et 355 al., 2018, 2014) reported strongest allocentric preference in temporal cortices, they attribute 356 mixed ego- and allocentric encoding for computing allo-to-ego conversions to human dorsal 357 premotor cortex (PMd), pre-supplementary motor area (pre-SMA) and precuneus, but not to 358 medial intraparietal sulcus (mIPS), the superior parietal lobe (SPL) or the superior parieto359 occipital (SPOC), which were predominantly egocentric (Chen et al., 2014; Chen and Crawford, 360 2020). Our data provides details at the single neuron level about mixed encoding and the 
transition from predominant allocentric encoding to egocentric encoding as soon as the reach goal is determined (Byrne et al., 2010), corroborating the predictions for dorsal premotor cortex from the human neuroimaging studies (Chen et al., 2018). Yet, different to what human imaging suggests, we see a marked similarity between PRR and PMd regarding allocentric encoding.

The relevance of allocentric, especially object-centered encoding in skeletomotor compared to oculomotor tasks was unclear so far, since detailed monkey neurophysiology data existed only for saccade tasks. The importance of object geometry for manual interaction with objects may explain why we found allocentric encoding while neurons in the lateral intraparietal area (LIP) did not show predominant object-centered encoding during an object-relative saccade task, which was otherwise similar to our task (Sabes et al., 2002). On the other hand, saccade studies in monkey parietal area 7a suggest that individual neurons can encode target location not only in gaze-centered (egocentric) reference frame (Andersen et al., 1985), but also show left-right selectivity for spatial information relative to task-relevant objects (Chafee et al., 2007; Crowe et al., 2008). Similarly, subsets of neurons in the supplementary eye fields (SEF) of the frontal cortex have been shown to be selective for the left or right end of an object or a pair of dots towards which a saccade is directed (Moorman and Olson, 2007; Olson et al., 2000; Olson and Gettner, 1999, 1995; Olson and Tremblay, 2000).

Yet, for binary left-right selectivity, it can be difficult to distinguish categorical rule-like encoding from an object-relative position code proper. Given that neurons, especially in the frontal areas, are known to represent categorical abstract rules (Wallis et al., 2001; Wallis and Miller, 2003), it was speculated if object-relative left-right neural selectivity could result from top-down rule signals in previous object-centered saccade experiments (Filimon, 2015). In our task, we sampled space-continuous selectivity profiles with five on-the-object positions. Rule encoding would predict identical selectivity profiles for object left/right/long/short conditions, which was not the case for most of the units we recorded. Instead, we observed a spectrum of ego- and object-centered encoding, including mixed reference frames and partial scaling, as typical for the computation of coordinate transformations in neural networks (Avillac et al., 2005; Brozovic et al., 2007; Deneve et al., 2001; Deneve and Pouget, 2003), which cannot be explained merely by rule encoding. We therefore interpret our observed neural selectivity 
391 allocentric representations of space in the context of skeletomotor planning, rather than 392 categorical rule encoding.

393 Remarkably, object-centered encoding was accompanied with size-invariant spatial encoding of 394 different on-the-object sites, at the single neuron and population levels. To our knowledge, this is 395 the first electrophysiology evidence showing that the fronto-parietal network utilizes size396 invariant positional code for movement planning.

397 Size-invariance is a coding property discussed in the context of object recognition and associated 398 with ventral stream processing (DiCarlo and Cox, 2007; Grill-Spector and Weiner, 2014; 399 Tacchetti et al., 2018). Yet, the relative position of object features is not just relevant for 400 recognition, but also for interaction with the object, e.g., to pick up different sized hammers 401 always at the end of their handle. Since we presented reference array and cue not simultaneously 402 (in one monkey), we consider it unlikely that the animal memorized the position information by 403 means of a visual pattern and that the size-invariant selectivity observed here "echoes" ventral 404 stream pattern encoding.

405 Previous studies showed generalized quantity encoding in neurons in the depth of the 406 intraparietal sulcus of monkeys (Tudusciuc and Nieder, 2007) and in parietal BOLD signals in 407 humans (Harvey et al., 2015), i.e., selectivity for quantity irrespective if quantity was presented 408 via the size of an object or the number of visual items. Due to the discrete nature of the on-the409 object cue positions, it could be that the animals memorized them via an object-relative (left410 most, middle, ...) or an abstracted positional code $\left(1^{\text {st }}, 2^{\text {nd }}, 3^{\text {rd }}, .\right.$. position on the object $)$ which is 411 numerical but still object-related. Translating back such abstracted numerical information into 412 spatial motor goal information would be a highly useful capacity, e.g., for foraging in discretized 413 environments (" 5 th tree from the left").

414 PRR and PMd are along the course of the dorsal visual processing pathway and we showed that 415 they express allocentric and size-invariant encoding with a short latency of a few hundred 416 milliseconds after presentation of an object-relative spatial cue. Allocentric and size invariant 417 abstract representations are two important properties which according to the classical two visual 418 pathways model (Goodale et al., 2004; Goodale and Westwood, 2004; Milner and Goodale, 419 2008) are associated with the areas along the ventral stream. The model suggests that in memory- 
guided actions, the dorsal stream could access the ventral stream neural codes through interaction with different areas in the ventral stream. However, it does not predict the latencies of the interaction and how quickly the codes could be accessible in the dorsal stream. If the model accounts for our results, as we had predicted in our earlier behavioral study (Taghizadeh and Gail, 2014), our findings strongly suggests that the interaction across the two pathways are almost immediate and does not take the long latencies which had been previously suggested by behavioral experiments (see (Westwood and Goodale, 2011) for review). Functional and direct and indirect anatomical connections between frontal and parietal cortex with areas of the ventral stream exist ((Borra et al., 2010), see reviews from (Cloutman, 2013; Perry and Fallah, 2014)). Our data suggest that in order to plan movements which take higher levels of computations and cognitive load for interacting with objects according to geometrical considerations, the two processing streams need to be functionally tightly connected and work together as a network to

432 support the dorsal stream for flexible action planning to interact with non-stationary dynamic 433 environments.

\section{Methods}

\section{$435 \quad$ Object-directed memory-guided reaching task}

436 The monkeys were seated in a primate chair in a dimly lit room in front of a fronto-parallel 437 touchscreen. With the help of head-fixation and trained gaze fixation (see below), the monkeys' 438 mid-sagittal plane and all egocentric references were aligned to the screen center. Visual stimuli 439 were presented on an LCD screen (19" ViewSonic VX922; onset latencies corrected; 440 background intensity of $0.16 \mathrm{~cd} / \mathrm{m} 2$ ) mounted behind the touchscreen (IntelliTouch, ELO 441 Systems, CA, USA). The distance between the monkeys' eyes and the screen was 39-45 $\mathrm{cm}$. 442 Throughout the text, conversions from centimeter to degree are based on a $40 \mathrm{~cm}$ distance.

443 The temporal structure of the task was identical in Exp-I and II (Fig. 1a). The monkey initiated a 444 trial by acquiring central gaze fixation (224 Hz CCD camera, ET-49B, Thomas Recording) and 445 hand fixation on the touchscreen. The gaze fixation stimulus was a filled red square of $0.5 \mathrm{~cm}$ $446\left(0.72^{\circ}\right)$ side length and $7 \mathrm{~cd} / \mathrm{m}^{2}$ intensity, and the hand fixation stimulus was a filled white square 447 of $0.5 \mathrm{~cm}\left(0.72^{\circ}\right)$ side length and $13 \mathrm{~cd} / \mathrm{m}^{2}$ intensity. Gaze and hand fixation was enforced within $448 \quad 2-3 \mathrm{~cm}\left(2.86-2.89^{\circ}\right)$ around each of the two immediately adjacent fixation points. In case of 
unsuccessful fixation, the trial was aborted and repeated at a random later time during the experiment.

451 Valid gaze and hand fixation for a random fixation period of 600-1100 ms was followed by a $452400 \mathrm{~ms}$ presentation of an array of five boxes, horizontally arranged and connected with a line 453 (reference object; details see below). The boxes indicated possible positions of the upcoming 454 spatial cue. The cue consisted of a small dot of $0.27 \mathrm{~cm}\left(0.39^{\circ}\right)$ diameter presented at the position 455 of one of the five reference object boxes. To balance task conditions, cue positions were selected 456 pseudo-randomly with increasing probability of so-far underrepresented conditions, and such 457 that the difference in number of correct repetitions between different positions did not exceed 458 three trials.

459 After an inter-stimulus interval (ISI) of $80 \mathrm{~ms}$ following the offset of the reference object, a 460 spatial cue was presented for $340 \mathrm{~ms}$. For monkey H, the ISI was removed and the reference 461 object remained visible during the $420(=80+340) \mathrm{ms}$ of cue presentation time. The cue 462 presentation was followed by a first variable memory period of 700-1200 ms for monkey K and 463 900-1200 ms for monkey $\mathrm{H}$, during which only the fixation stimuli were shown (visual memory). 464 After this delay, a reach object of the same type as the reference object was presented. The 465 monkey was instructed to later touch the box on the reach object which corresponded to the box 466 that was cued on the reference object, e.g., for a cue seen at the left-most box of the reference 467 object, the monkey should reach towards the left-most box of the reach object (target), 468 irrespective of the absolute position of the reach object on the screen. The onset of the reach 469 object was followed by a second delay period of 615-646 ms during which the reach object was 470 visible but the monkey was required to maintain gaze fixation and to withhold the movement 471 (movement planning). Continued visibility of the reach object allowed the animals mentally 472 maintaining the reach goal location either in an object-centered or egocentric reference frame. 473 After the second delay period, the hand fixation stimulus disappeared. This served as the GO cue 474 to reach to and touch the cued on-the-object target location within $1000 \mathrm{~ms}$ while holding central 475 ocular fixation. Reach endpoints had to be within an elliptical area (horizontal semi-minor axis $4761.2 \mathrm{~cm}\left(1.72^{\circ}\right)$; vertical semi-major axis $4 \mathrm{~cm}\left(5.71^{\circ}\right)$ ) around the target box. After holding the 477 target for $220 \mathrm{~ms}$ (monkey H; 300ms monkey K) the trial counted as successful and the monkey 478 received a visual (a small, light gray dot of $0.27 \mathrm{~cm}\left(0.39^{\circ}\right)$ diameter on the target box of the 
reach object), an acoustic (a high-pitched tone) feedback, and a drop of juice as reward. The monkey's mid-sagittal plane, gaze and hand fixation points aligned to the center of the screen. The reference object randomly presented at one of two egocentric locations, left or right of the screen center, with equal eccentricity (Fig. 1b). The reach object also presented either left or right, but the possible locations differed across experiments I and II (see below). Here and throughout the text, "location" of the object refers to the position of the center of mass of the object on the screen, i.e., the center point of the central box on the object. Reference object, reach object and cue had a low intensity gray tone $\left(2 \mathrm{~cd} / \mathrm{m}^{2}\right)$.

Exp-1 Position invariance In Exp-I, reference and reach objects where always identical in terms of visual appearance. The individual boxes of the reference and reach objects were $0.35 \mathrm{~cm}$ $\left(0.50^{\circ}\right)$ squares with $2.8 \mathrm{~cm}\left(4.00^{\circ}\right)$ center-to-center distance. In terms of location, reference and reach object either matched (position-congruent trials) or differed (position-incongruent). Left and right screen locations were $(\mathrm{x}, \mathrm{y})=( \pm 1.4,2.7) \mathrm{cm}=( \pm 2.0,3.8)^{\circ}$ relative to screen center. This means, objects were vertically elevated above the eye and hand fixation position to prevent visual interference with fixation stimuli and obstruction by the animal's arm. Horizontally, in position-incongruent trials, the locations of the reference and reach object boxes were set off by one box distance, such that four out of five box positions overlapped between reference (potential cue locations) and reach object (potential reach targets). For example, boxes 2 to 5 (counting from left to right) of a left-side object had identical egocentric locations to boxes 1 to 4 of a right-side object (Fig. 1b). Therefore, while the cue (target) could take five different positions relative to the reference (decision) object, i.e., five different object-centered positions, they covered in total six different potential egocentric locations on the screen. The 20 different combinations of cue, reference and reach object positions $(5 \times 2 \times 2)$ were presented pseudorandomly (algorithm as above). By the nature of the behavioral task, the cue and the reach goal always had the same object-centered position. In position-congruent trials, the cue and the reach goal additionally had the same egocentric locations. In position-incongruent trials, the cue and the reach goal differed in egocentric position and the reach goal needed to be determined based on the object-based location of the target box. Since the congruency of the trials was unpredictable, the monkey was encouraged to follow object-centered encoding of the cue in all trials for successful task performance. The monkeys could only determine the egocentric reach goal location upon occurrence of the reach object. 
510 Exp-2-Size invariance In Exp-II, reference and reach objects always differed in terms of location 511 (position-incongruent trials only) and could additionally differ in size. In long-object trials (size512 congruent, Fig. 1c, top left), the reference object was identical to Exp-I. In short-object trials

513 (size-incongruent, Fig. 1c, top right), the boxes of the reference object were only $1.4 \mathrm{~cm}\left(2.00^{\circ}\right)$ 514 apart, i.e. half the spacing of the long object. The reach object in both long and short trials was 515 identical to the long reference object. Both long and short reference objects were presented at the 516 same left and right locations as in Exp-I. Unlike Exp-I, the reach object in Exp-II was always 517 position-incongruent to the reference object. While the reference and reach object could be 518 congruent or incongruent in terms of horizontal location (as in Exp-I), the reach object always 519 had a vertical position offset and was located at $(x, y)=( \pm 2.3,4.4) \mathrm{cm}=( \pm 3.4,6.3)^{\circ}($ Fig. 1c, 520 bottom). This offset was introduced to maximally encourage object-based encoding of the cue 521 location during the visual memory period, since the purpose of Exp-II was to specifically test for 522 scale invariance during object centered encoding. Again, by nature of the task, the cue and the 523 reach goal always had the same object-centered position. Long- and short-object trials were 524 presented in alternating blocks. In long-object blocks, as in Exp-I, all 20 different combinations 525 of cue, reference and reach object positions $(5 \times 2 \times 2)$ were pseudo-randomly presented. In short526 object blocks, reference object left and right conditions were blocked for ease of performance. In 527 each sub-block, the 5x2 combinations of cue and reach object locations where randomly 528 interleaved such that horizontal (in-)congruency was always unpredictable and hence the reach 529 goal unknown prior to onset of the reach object.

530 Behavioral success rate was calculated as percentage of correctly performed reaches relative to 531 initiated trials in which the monkey continued until after he started the reach movement.

532 Single unit selection. In both experiments, only "active" units were included in the reference 533 frame analysis and were defined as units which on average (across all trials, regardless of task 534 condition) fired at least 5 spikes within a time interval extending from 100 ms before until 1500 $535 \mathrm{~ms}$ after cue onset in correctly performed trials. Average firing rate across all selected units in 536 Exp-I for PRR and PMd was 15.63 and 15.02, respectively, in monkey K; 12.03 and 10.66 in 537 monkey H. In Exp-II for PRR and PMd it was 19.1 and 15.7 in monkey K, and 10.6 and 11.6 in 538 monkey $\mathrm{H}$. 
based on single units' firing rates At the single unit level, we distinguished object-centered and egocentric reference frames by comparing each neuron's spatial selectivity profile for the five cue stimulus positions in trials when an object was presented on the left side with the trials when the corresponding object was presented on the right side. During visual memory, we compared the reference object positions, irrespective of reach object position. Vice versa, during movement planning we compared the reach object positions, irrespective of reference object position. The object-centered hypothesis predicts object-left and object-right selectivity profiles to be identical in shape, but shifted relative to each other in egocentric screen space (Fig. 2a-c, left). The egocentric hypothesis instead predicts the selectivity profiles to be identical only in the range of object compartments that overlap in egocentric screen space (Fig. 2a-c, right). For each cell, we measured the similarity of the two selectivity profiles to quantify how well either hypothesis can explain the response of the neuron. We used the Pearson's linear correlation coefficient, similar to (Mullette-Gillman et al., 2005), between each pair of the selectivity profiles, i.e., reference object left versus right; reach object left versus right. For variance-stabilization, the bounded correlation coefficients were subjected to Fisher's z transformation (inverse hyperbolic tangent function) before using them for any further analysis and statistical tests. For brevity, we refer to the Fisher z-transformed correlation coefficients as "correlation coefficients".

557 Since the correlation coefficient is insensitive to linear scaling, the similarity between the shapes of the selectivity profiles is quantified independent of potential gain modulation effects on the neural firing rates. This means, if object location modulates responses to all cue positions by an equal factor, without affecting relative response strengths for different on-the-object cue positions, the correlation method considers this outcome to be in accordance with the objectcentered hypothesis. Our complementary decoding method (see below) is sensitive to gain 563 effects on neural activity.

564 For each cell and pairwise selectivity profile, the correlation coefficient was calculated twice, 565 once in each reference frame. The object-centered correlation (objCorr) is the correlation 566 coefficient between the samples of the object left and right selectivity profiles with 567 corresponding object-centered cue locations. (Fig. 2a, left, green arrows show points with 568 equivalent object-centered location). The egocentric correlation (egoCorr) is the correlation 
coefficient between samples of the object left and right tuning curves with corresponding egocentric locations. (Fig. 2a, right, dark red arrows show points with equivalent egocentric location). Each of the correlation values provides a measure that determines the validity of the

572 corresponding hypothesis for each cell's activity profile. The difference objCorr-egoCorr 573 between them indicates the neuron's preference of reference frame. We will refer to this 574 difference as Position Invariance (PI). Positive values indicate selectivity profile of the neuron 575 was better explained by the object-centered hypothesis (= invariance with respect to egocentric 576 location), while negative values point towards the egocentric hypothesis (= invariance with respect to object-centered location).

578 In order to reduce effect of outliers in trial-by-trial firing rates, we estimated the mean firing rate 579 for every unit with a bootstrap method (200x resampling; bootstrp() function in MATLAB) and 580 the PI was calculated for every bootstrap sample. In addition, for every bootstrap sample, the null 581 distribution of PI was estimated by randomly shuffling trials (trials used to generate this 582 bootstrap sample) 100 times, across all task conditions, and the average null-PI was subtracted 583 from the corresponding PI.

584 Visual memory vs movement planning reference frame: To study changes in the preferred 585 reference frame across time, for each cell PI was calculated in $300 \mathrm{~ms}$ windows, sliding by 50 $\mathrm{ms}$, extending from $600 \mathrm{~ms}$ before until $600 \mathrm{~ms}$ after reach object onset (data was aligned to reach object onset). In all time bins earlier than reach object onset (up to bin [-150 150] ms), selectivity profiles were computed for cue relative to reference object (memory period); after reach object onset, selectivity profiles were computed for target relative to reach object

590 (movement planning period). The time bin centered on reach object onset ([-150 150]ms) was

591 included in both memory and movement planning periods.

592 When comparing PI in visual memory versus movement planning, we tested across the 593 population of neurons, if a strong preference for one reference frame during visual memory 594 would be associated with a strong preference for the same, the other, or no preferred reference 595 frame during movement planning. For this, we plotted the PI of each neuron in memory vs 596 planning period on orthogonal axes (Fig. 3b), and measured the angular deviation $\theta$ from the $\mathrm{x}+$ 597 axis, between -180 and 180 degrees, corresponding to the arctan of the ratio 598 PI_planning/PI_memory. Neurons showing a preferred reference frame selectively in only one 
delay period fall onto one of the main axes, neurons with strongly (anti-) correlated preference

600 fall on the diagonals. Predominant subpopulations of either type would result in an

601 inhomogeneous distribution of the angles. Distributions were estimated by binning the data into

$60245^{\circ}$ bins, and subjected to Hartigan's dip test for multimodality. We compared responses $300 \mathrm{~ms}$

603 preceding (late memory) and 300-600 ms after (late planning period) reach object onset, when

604 the neural activity is comparably stable after the transient change in stimulus.

605 Scaling of the spatial selectivity to the object size in Exp-II: Exp-II aimed at further 606 characterizing object-centered encoding. Therefore, we analyzed firing rates only during the late 607 memory period when neurons have reached their sustained activity and predominant position608 invariant (object-centered) preference was expected based on the results of Exp-I. The object609 centered hypothesis predicts that selectivity profiles should scale with the object size in the 610 egocentric screen space (Fig. 4a, left), independent of its location, meaning similar object611 centered selectivity profiles in all four conditions of object size and location. The egocentric 612 hypothesis predicts that selectivity profiles for small object size should resample part of the 613 selectivity profile for large object size in the egocentric screen space (Fig. 4a, right). To test this, 614 we quantified two correlation coefficients for every neuron: alloCorr was the correlation 615 coefficient between a neuron's activity in ten conditions ( 5 on-the-object cue positions times 2 616 reference object locations) in long-object trials and their corresponding conditions (with identical 617 object-centered cue location) in short-object trials (2 sets of 10 green arrows in Supplementary 618 Fig. 1a). egoCorr was the correlation coefficient between neuron's activity in six conditions (3 619 on-object cue positions times 2 reference object locations) in long-object trials and their 620 corresponding conditions (with identical egocentric locations) in short-object trials (2 sets of 6 621 red arrows in Supplementary Fig. 1b). To account for both size and position invariance at the 622 same time, alloCorr and egoCorr were calculated across all possible pairing of the firing rates 623 between the short and long trials, regardless of object location. We calculated alloCorr-egoCorr 624 to quantify how far a neuron complies with the prediction of object-centered Position and Size 625 Invariant (PSI) encoding, and refer to this measure as PSI. In the same way as in Exp-I, PSI for 626 every cell was bootstrapped and a shuffle predictor subtracted.

627 Decoding population activity. As an additional population-level analysis, we used a cross628 conditional decoding approach: A 5-way classifier for decoding cue or target position on the 
object was trained with data from one condition (e.g. reference object located left on screen) and

630 its performance was tested on data from another condition (reference object right). For Exp-I,

631 there are two conditions, object-left and object-right, in the memory and planning period,

632 respectively. A decoder that is only trained on trials of the object-left condition but accurately

633 predicts the position in object-right condition trials (left-right generalization) suggests a

634 predominant position-invariant (object-centered) encoding in the data. This will show as a

635 confusion matrix where only the main diagonal is populated (Fig. 3d). On the other hand, a

636 systematic offset in the predicted labels, e.g., object-right trials at positions 1, 2, 3, 4 (counting

637 the boxes from left to right) are decoded as positions 2, 3, 4, 5 by the object-left classifier, is

638 indicative of an egocentric encoding, because these positions correspond to the same egocentric

639 locations. Such pattern would show as a confusion matrix populated along the secondary

640 diagonal. In Exp-II, there are four conditions, long-object-left, long-object-right, short-object-

641 left, short-object-right, and each pair has to be compared independently. To quantify the size-

642 invariance of the neural population, we compared each long-with each short-object condition,

643 resulting in four comparisons.

644 For the decoding analysis, we included only units for which we recorded at least 15 trials per

645 cue/target position in the training condition. This enabled us to select a random subset of 15 trials

646 per position of the training condition from each unit. The average firing rates of the selected

647 units in a 300ms window during the selected trials were combined to form the 75 feature vectors

648 for training the decoder. Feature vectors for testing were built similarly by combining a random

649 subset of as many trials as possible (same number for each position) of the testing condition from

650 the same units. This randomization was repeated 1000 times, each time choosing random subsets

651 of trials from each unit. This way, we can include the whole dataset in the decoding analysis,

652 despite each unit being recorded for a different number of trials. For each repetition, an

653 independent classifier was trained. We used an error-correcting output codes (ECOC) model

654 with 10 binary linear support vector machines as the classifier (MATLAB function: fitcecoc). To

655 get a time-continuous estimate, we repeated the analysis, each time shifting the window by

$65650 \mathrm{~ms}$. During the visual memory period, the condition of each trial was defined by the reference

657 object, during the movement planning period it was defined by the reach object. For the time

658 windows that overlapped with both periods (around reach object onset), the condition was 
defined by the period with the most overlap. For the window centered on the reach object onset,

660 both definitions where used.

661 In order to quantify the dominance of a reference frame, we calculate the difference $p_{o b j}-p_{\text {ego }}$

662 in percentage of trials decoded in accordance with the object-centered hypothesis and percentage

663 of trials decoded in accordance with the egocentric hypothesis. This measure will be referred to 664 as Reference Frame Difference (RFD). A value of 1 shows complete object-centered encoding, 665 while a value of -1 shows complete egocentric encoding. For this calculation, we only consider 666 the test trials to positions that have an egocentric equivalent in the training condition. For 667 example, we cannot include test trials to the right-most position when the object is on the right, 668 because this location does not exist in the object-left condition (Fig. 1a and Fig. 3d). The 669 number of eligible positions depends on the conditions that are being compared: In Exp-I, four 670 positions overlap (Fig. 3d) while for comparisons of short and long objects in Exp-II, two 671 positions can be considered (three positions overlap, but one of these is the same in both 672 reference frames and hence does not help to distinguish them, Supplementary Fig. 1).

673 To test for a significant difference from zero, we calculate the RFD for each of the 1000 674 randomizations and compute the percentage of values lying below and above 0 . If one of these 675 percentages is less than half the alpha criterion, it is considered significant.

676 In all analysis where results of statistical tests were compared across multiple time bins, the 677 significance level was adjusted to account for multiple comparisons by correcting for the false678 discovery rate, allowing a proportion of false positives of less than $5 \%$.

679 Animal implantation and neural recordings The procedures for animal preparation and neural 680 recordings were described previously (Westendorff et al., 2010) and are here repeated for 681 completeness. Numerical values have been adjusted to the current experiment. Two monkeys 682 implanted with a titanium head holder and two magnetic resonance imaging (MRI)-compatible 683 recording chambers, custom-fit to the monkeys' heads (3di, Jena Germany; and Thomas 684 Recording, Giessen, Germany). Chamber positioning above PRR (Horsley Clarke coordinates: $68510 \mathrm{~mm}$ contralateral and $13 \mathrm{~mm}$ posterior for monkey $\mathrm{K} ; 12.5 \mathrm{~mm}$ contralateral and $13.5 \mathrm{~mm}$ 686 posterior for monkey $\mathrm{H}$ ) and $\mathrm{PMd}(18.5 \mathrm{~mm}$ contralateral and $20 \mathrm{~mm}$ anterior for monkey K; 19 mm contralateral and $22 \mathrm{~mm}$ anterior for monkey H) was guided by pre-surgical structural MRI 
688 and confirmed by postsurgical MRI. Sustained direction-selective neural responses during 689 center-out reach planning (memory period) served as physiological signature to confirm the

690 region of interest in both areas. Both chambers implanted contralaterally to the handedness of the 691 monkey (left hemisphere). All surgical and imaging procedures were conducted under general 692 anesthesia. We used two five-channel microdrives ("mini-matrix"; Thomas Recording) for 693 extracellular recordings, mostly simultaneously in both chambers. The raw signals of the 694 electrodes were pre-amplified (20x; Thomas Recording), band pass filtered, and amplified (154 $695 \mathrm{~Hz}$ to $8.8 \mathrm{kHz}$; 400-800x; Plexon) before online spike sorting was conducted (Sort Client; 696 Plexon). Spike times and spike waveforms were recorded and later subjected to additional offline 697 sorting (Offline Sorter; Plexon).

698 Both animals were housed in social groups with one or two male conspecifics in facilities of the 699 German Primate Center. The facilities provide cage sizes exceeding the requirements by German 700 and European regulations, and access to an enriched environment including wooden structures 701 and various toys. All procedures have been approved by the responsible regional government 702 office [Niedersächsisches Landesamt für Verbraucherschutz und Lebensmittelsicherheit 703 (LAVES)] under permit numbers 3392 42502-04-13/1100 and comply with German Law and the 704 European Directive 2010/63/EU regulating use of animals in research.

\section{Acknowledgements}

706 We thank Sina Plümer for help with data collection and technical support, Klaus Heisig for help 707 with setup maintenance, Leonore Burchardt for help with animal training and Dirk Prüße for 708 technical support.

709 This work was supported by and benefited from the State of Lower Saxony (grant VWZN2563), 710 the European Commission in the context of the Plan4Act consortium (http://plan4act-project.eu; 711 EC-H2020-FETPROACT-16732266), and the German Research Foundation in the context of the 712 Collaborative Research Center Cognition of Interaction (DFG SFB-1528).

\section{References}

714 Aagten-Murphy, D., Bays, P.M., 2019. Independent working memory resources for egocentric 715 and allocentric spatial information. PLoS Comput. Biol. 15, e1006563. 
Aglioti, S., DeSouza, J.F., Goodale, M.A., 1995. Size-contrast illusions deceive the eye but not the hand. Curr. Biol. 5, 679-685. https://doi.org/10.1016/s0960-9822(95)00133-3

Andersen, R.A., Essick, G.K., Siegel, R.M., 1985. Encoding of spatial location by posterior parietal neurons. Science (80-. ). 230, 456-458.

Avillac, M., Deneve, S., Olivier, E., Pouget, A., Duhamel, J.R., 2005. Reference frames for representing visual and tactile locations in parietal cortex. Nat.Neurosci. 8, 941-949.

Batista, A.P., Buneo, C.A., Snyder, L.H., Andersen, R.A., 1999. Reach plans in eye-centered coordinates. Science (80-. ). 285, 257-260. frames for reach planning in macaque dorsal premotor cortex. J.Neurophysiol. 98, 966-983.

Battaglia-Mayer, A., Caminiti, R., Lacquaniti, F., Zago, M., 2003. Multiple levels of representation of reaching in the parieto-frontal network. Cereb.Cortex 13, 1009-1022.

Bernier, P.M., Grafton, S.T., 2010. Human posterior parietal cortex flexibly determines reference frames for reaching based on sensory context. Neuron 68, 776-788.

Borra, E., Ichinohe, N., Sato, T., Tanifuji, M., Rockland, K.S., 2010. Cortical connections to area TE in monkey: hybrid modular and distributed organization. Cereb. Cortex 20, 257-270. https://doi.org/10.1093/cercor/bhp096

Bremner, L.R., Andersen, R.A., 2014. Temporal Analysis of Reference Frames in Parietal Cortex Area 5d during Reach Planning. J.Neurosci. 34, 5273-5284.

Bremner, L.R., Andersen, R.A., 2012. Coding of the reach vector in parietal area 5d. Neuron 75, $342-351$. visuomotor transformations. J.Neurosci. 27, 10588-10596. 
transformations for reaching. Nature 416, 632-636.

742 Burgess, N., 2006. Spatial memory: how egocentric and allocentric combine. Trends Cogn Sci. $10,551-557$.

Byrne, P.A., Cappadocia, D.C., Crawford, J.D., 2010. Interactions between gaze-centered and allocentric representations of reach target location in the presence of spatial updating. Vis.

Caminiti, R., Innocenti, G.M., Battaglia-Mayer, A., 2015. Organization and evolution of parietofrontal processing streams in macaque monkeys and humans. Neurosci. Biobehav. Rev. 56, 73-96. https://doi.org/10.1016/j.neubiorev.2015.06.014

Caminiti, R., Johnson, P.B., Burnod, Y., Galli, C., Ferraina, S., 1990. Shift of preferred directions of premotor cortical cells with arm movements performed across the workspace. Exp.Brain Res. 83, 228-232.

Caminiti, R., Johnson, P.B., Galli, C., Ferraina, S., Burnod, Y., 1991. Making arm movements

Chafee, M. V, Averbeck, B.B., Crowe, D.A., 2007. Representing spatial relationships in posterior parietal cortex: single neurons code object-referenced position. Cereb.Cortex 17,

Chang, S.W., Snyder, L.H., 2012. The representations of reach endpoints in posterior parietal cortex depend on which hand does the reaching. J.Neurophysiol. 107, 2352-2365.

Chang, S.W., Snyder, L.H., 2010. Idiosyncratic and systematic aspects of spatial representations in the macaque parietal cortex. Proc.Natl.Acad.Sci.U.S.A 107, 7951-7956.

Chen, Y., Byrne, P., Crawford, J.D., 2011. Time course of allocentric decay, egocentric decay, and allocentric-to-egocentric conversion in memory-guided reach. Neuropsychologia 49, 49-60.

Chen, Y., Crawford, J.D., 2020. Allocentric representations for target memory and reaching in 
human cortex. Ann. N. Y. Acad. Sci. 1464, 142-155.

https://doi.org/https://doi.org/10.1111/nyas.14261

Chen, Y., Monaco, S., Byrne, P., Yan, X., Henriques, D.Y., Crawford, J.D., 2014. Allocentric versus Egocentric Representation of Remembered Reach Targets in Human Cortex. J.Neurosci. 34, 12515-12526.

772 Chen, Y., Monaco, S., Crawford, J.D., 2018. Neural substrates for allocentric-to-egocentric conversion of remembered reach targets in humans. Eur. J. Neurosci. 47, 901-917. https://doi.org/10.1111/ejn.13885

Cisek, P., Kalaska, J.F., 2005. Neural correlates of reaching decisions in dorsal premotor cortex:

Cloutman, L.L., 2013. Interaction between dorsal and ventral processing streams: Where, when specification of multiple direction choices and final selection of action. Neuron 45, 801814.

Cohen, Y.E., Andersen, R.A., 2002. A common reference frame for movement plans in the posterior parietal cortex. Nat.Rev.Neurosci. 3, 553-562.

Committeri, G., Galati, G., Paradis, A.L., Pizzamiglio, L., Berthoz, A., LeBihan, D., 2004.

Crammond, D.J., Kalaska, J.F., 1994. Modulation of preparatory neuronal activity in dorsal premotor cortex due to stimulus-response compatibility. J.Neurophysiol. 71, 1281-1284.

Crowe, D.A., Averbeck, B.B., Chafee, M. V, 2008. Neural ensemble decoding reveals a correlate of viewer- to object-centered spatial transformation in monkey parietal cortex. J.Neurosci. $28,5218-5228$.

Cui, H., Andersen, R.A., 2011. Different representations of potential and selected motor plans by distinct parietal areas. J.Neurosci. 31, 18130-18136.

Deneve, S., Latham, P.E., Pouget, A., 2001. Efficient computation and cue integration with noisy 
population codes. Nat.Neurosci. 4, 826-831.

Deneve, S., Pouget, A., 2003. Basis functions for object-centered representations. Neuron 37, 347-359. https://doi.org/10.1016/s0896-6273(02)01184-4

DiCarlo, J.J., Cox, D.D., 2007. Untangling invariant object recognition. Trends Cogn. Sci. 11, 333-341. https://doi.org/10.1016/j.tics.2007.06.010

Filimon, F., 2015. Are All Spatial Reference Frames Egocentric? Reinterpreting Evidence for Allocentric, Object-Centered, or World-Centered Reference Frames. Front. Hum. Neurosci. 9, 648. https://doi.org/10.3389/fnhum.2015.00648

Gail, A., Andersen, R.A., 2006. Neural dynamics in monkey parietal reach region reflect context-specific sensorimotor transformations. J.Neurosci. 26, 9376-9384.

Galati, G., Lobel, E., Vallar, G., Berthoz, A., Pizzamiglio, L., Le Bihan, D., 2000. The neural basis of egocentric and allocentric coding of space in humans: a functional magnetic resonance study. Exp.Brain Res. 133, 156-164.

Goodale, M.A., Westwood, D.A., 2004. An evolving view of duplex vision: separate but

811 Goodale, M.A., Westwood, D.A., Milner, A.D., 2004. Two distinct modes of control for objectdirected action. Prog.Brain Res. 144, 131-144.

813 Grill-Spector, K., Weiner, K.S., 2014. The functional architecture of the ventral temporal cortex and its role in categorization. Nat. Rev. Neurosci. 15, 536-548.

816 Harvey, B.M., Fracasso, A., Petridou, N., Dumoulin, S.O., 2015. Topographic representations of object size and relationships with numerosity reveal generalized quantity processing in human parietal cortex. Proc. Natl. Acad. Sci. U. S. A. 112, 13525-13530. 
Hwang, E.J., Andersen, R.A., 2012. Spiking and LFP activity in PRR during symbolically instructed reaches. J.Neurophysiol. 107, 836-849.

Johnson, P.B., Ferraina, S., Bianchi, L., Caminiti, R., 1996. Cortical networks for visual reaching: physiological and anatomical organization of frontal and parietal lobe arm regions. Cereb.Cortex 6, 102-119.

Marzocchi, N., Breveglieri, R., Galletti, C., Fattori, P., 2008. Reaching activity in parietal area V6A of macaque: eye influence on arm activity or retinocentric coding of reaching movements? Eur.J.Neurosci. 27, 775-789.

McGuire, L.M., Sabes, P.N., 2011. Heterogeneous representations in the superior parietal lobule are common across reaches to visual and proprioceptive targets. J.Neurosci. 31, 6661-6673.

Milner, A.D., Goodale, M.A., 2008. Two visual systems re-viewed. Neuropsychologia 46, 774785.

Moorman, D.E., Olson, C.R., 2007. Impact of experience on the representation of objectcentered space in the macaque supplementary eye field. J.Neurophysiol. 97, 2159-2173.

Moser, E.I., Kropff, E., Moser, M.-B., 2008. Place cells, grid cells, and the brain's spatial representation system. Annu. Rev. Neurosci. 31, 69-89.

Mullette-Gillman, O.A., Cohen, Y.E., Groh, J.M., 2005. Eye-centered, head-centered, and complex coding of visual and auditory targets in the intraparietal sulcus. J.Neurophysiol. 94,

Neggers, S.F., Van der Lubbe, R.H., Ramsey, N.F., Postma, A., 2006. Interactions between egoand allocentric neuronal representations of space. Neuroimage. 31, 320-331. movements regardless of the visual attributes of instructional cues. J.Neurophysiol. 81, 2340-2346. 
845

Olson, C.R., Gettner, S.N., 1995. Object-centered direction selectivity in the macaque supplementary eye field. Science (80-. ). 269, 985-988.

Olson, C.R., Gettner, S.N., Ventura, V., Carta, R., Kass, R.E., 2000. Neuronal activity in macaque supplementary eye field during planning of saccades in response to pattern and spatial cues. J.Neurophysiol. 84, 1369-1384.

Olson, C.R., Tremblay, L., 2000. Macaque supplementary eye field neurons encode objectcentered locations relative to both continuous and discontinuous objects. J.Neurophysiol. 83, 2392-2411.

Perry, C.J., Fallah, M., 2014. Feature integration and object representations along the dorsal stream visual hierarchy. Front. Comput. Neurosci. 8, 84. https://doi.org/10.3389/fncom.2014.00084

Pesaran, B., Nelson, M.J., Andersen, R.A., 2006. Dorsal premotor neurons encode the relative position of the hand, eye, and goal during reach planning. Neuron 51, 125-134.

Riehle, A., Requin, J., 1989. Monkey primary motor and premotor cortex: single-cell activity related to prior information about direction and extent of an intended movement. J. Neurophysiol. 61, 534-549. https://doi.org/10.1152/jn.1989.61.3.534

Sabes, P.N., Breznen, B., Andersen, R.A., 2002. Parietal representation of object-based saccades. J.Neurophysiol. 88, 1815-1829.

Snyder, L.H., Batista, A.P., Andersen, R.A., 1997. Coding of intention in the posterior parietal cortex. Nature 386, 167-170.

Tacchetti, A., Isik, L., Poggio, T.A., 2018. Invariant Recognition Shapes Neural Representations of Visual Input. Annu. Rev. Vis. Sci. 4, 403-422. https://doi.org/10.1146/annurev-vision091517-034103

Taghizadeh, B., Gail, A., 2014. Spatial task context makes short-latency reaches prone to induced Roelofs illusion. Front Hum.Neurosci. 8, 673.

Thaler, L., Goodale, M.A., 2011a. The role of online visual feedback for the control of target- 
directed and allocentric hand movements. J.Neurophysiol. 105, 846-859.

872 Thaler, L., Goodale, M.A., 2011b. Reaction times for allocentric movements are 35 ms slower 873 than reaction times for target-directed movements. Exp.Brain Res. 211, 313-328.

874 Thaler, L., Goodale, M.A., 2011c. Neural substrates of visual spatial coding and visual feedback control for hand movements in allocentric and target-directed tasks. Front Hum.Neurosci. 5,

Tudusciuc, O., Nieder, A., 2007. Neuronal population coding of continuous and discrete quantity in the primate posterior parietal cortex. Proc. Natl. Acad. Sci. U. S. A. 104, 14513-14518. https://doi.org/10.1073/pnas.0705495104

Vallar, G., Lobel, E., Galati, G., Berthoz, A., Pizzamiglio, L., Le, B.D., 1999. A fronto-parietal

Wallis, J.D., Anderson, K.C., Miller, E.K., 2001. Single neurons in prefrontal cortex encode

Wallis, J.D., Miller, E.K., 2003. From rule to response: neuronal processes in the premotor and abstract rules. Nature 411, 953-956. https://doi.org/10.1038/35082081

887 Westendorff, S., Klaes, C., Gail, A., 2010. The cortical timeline for deciding on reach motor goals. J.Neurosci. 30, 5426-5436.

Westwood, D.A., Goodale, M.A., 2011. Converging evidence for diverging pathways: neuropsychology and psychophysics tell the same story. Vis. Res. 51, 804-811.

891 Wise, S.P., Boussaoud, D., Johnson, P.B., Caminiti, R., 1997. Premotor and parietal cortex: corticocortical connectivity and combinatorial computations. Annu.Rev.Neurosci. 20, 2542.

894 Zaehle, T., Jordan, K., Wustenberg, T., Baudewig, J., Dechent, P., Mast, F.W., 2007. The neural basis of the egocentric and allocentric spatial frame of reference. Brain Res. 1137, 92-103. 
899 Zipser, D., Andersen, R.A., 1988. A back-propagation programmed network that simulates

900 response properties of a subset of posterior parietal neurons. Nature 331, 679-684.

901 https://doi.org/10.1038/331679a0

902

903

904

905

906

907

908

909

910

911

912

913

914

915

916

917

918 


\section{Supplementary Figure 1 Corresponding position information for calculating Position and Size Invariance (PSI) between different task conditions}

(a)

Object-centered size and position invariance

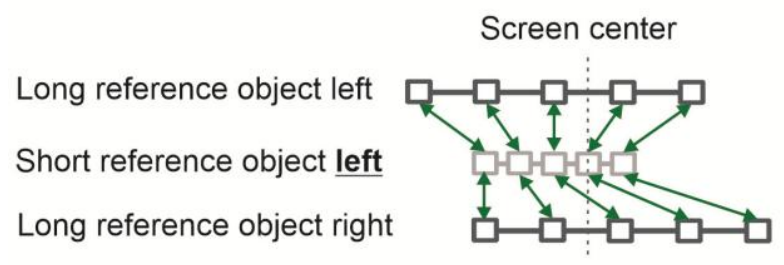

Screen center

Long reference object left

Short reference object right

Long reference object right (b)

Egocentric

Screen center

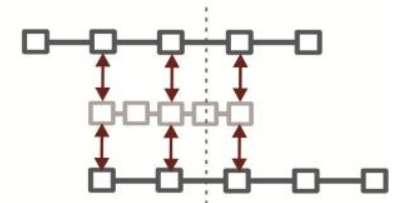

Screen center

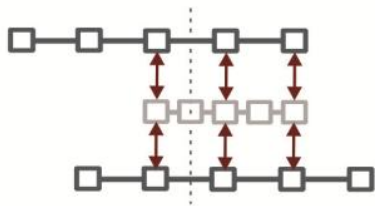

(a) Corresponding positions for calculating alloCorr. Invariance with respect to object position and object size predicts similar neural selectivity profiles between short-object and long-object conditions when the profile is computed relative to the within-object numerical box positions $(1,2,3, \ldots)$. To calculate alloCorr, we considered all possible correspondences (green arrows) between long and short objects when the short objects presented on the left (top panels) or right (bottom row) of the screen center (vertical dotted line). Box $1(2,3, \ldots)$ of the object here always corresponds to box $1(2,3, \ldots)$, irrespective of object position and size. We correlated a vector of 20 firing rates from long-object selectivity profiles with a vector of 20 firing rates from short-object profiles. Higher alloCorr results in higher PSI. (b) Corresponding positions for calculating egoCorr. The egocentric hypothesis predicts similar activity profiles for boxes between long and short objects which share the same position on the screen (= relative to the body). Accordingly, to calculate egoCorr, we considered every possible overlap (red arrows) between long and short objects when objects presented on the left (top row) or right (bottom row) of the screen center (vertical dotted line). We correlated a vector of 10 firing rates from long-object selectivity profiles with a vector of 10 firing rates from short-object profiles. Higher egoCorr results in lower PSI. 


\section{Supplementary Figure 2 Example unit with mixed reference frame in Exp II}

(a)

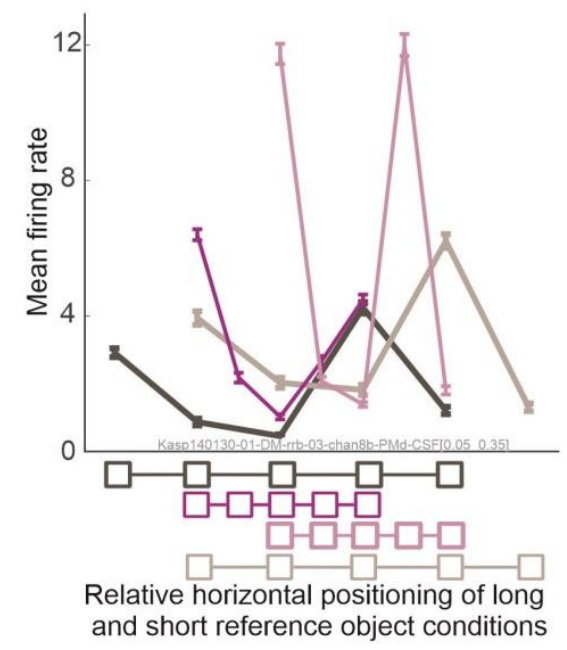

(b)

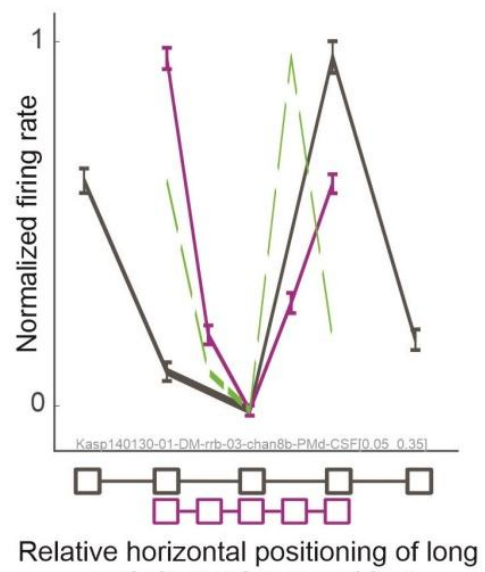
and short reference object (c)

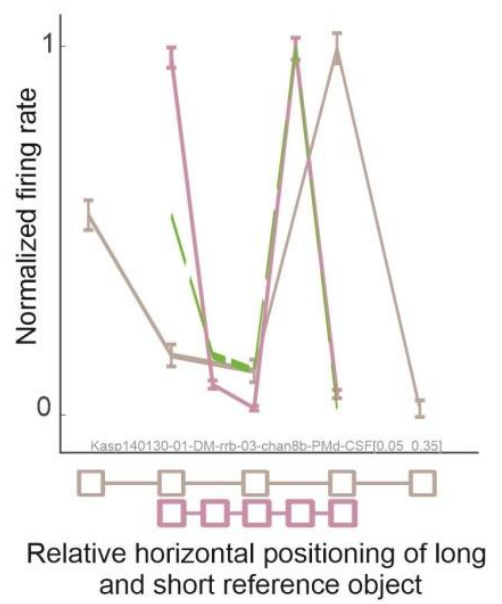

(a) Selectivity profiles of a PMd unit in early memory period, 50 - $350 \mathrm{~ms}$ after cue offset, for different object size and positions. (b) and (c) show normalized version of the same selectivity profiles as in (a), separately for long and short objects left (b) and right (c). The green dashed curves show the predicted selectivity profile for the short object trials based on the based on the selectivity profiles in the long object trials, assuming ideal object-centered encoding (i.e., the squeezed version of the long object selectivity profiles relative to the central box of the object). For this unit selectivity profiles in long-object-left (dark grey) and long-object-right (light grey) indicate position invariance. Also, the short-object-right (light purple) selectivity profile is quite close to a horizontally scaled version of the long-object profile, with gain modulation. In this sense, this unit meets predictions of a position- and size-invariant object-centered reference frame. However, the short-object-left profile deviates from the prediction, suggesting some form of mixed reference frame. 